\title{
AVALIAÇÕES PRELIMINARES DA TOXICIDADE AGUDA DO PESTICIDA FIPRONIL AOS ROTÍFEROS ASPLANCHNA SIEBOLDI, BRACHIONUS CAUDATUS E LEPADELLA PATELLA.
}

Raquel Aparecida Moreira ${ }^{1}$

\author{
Renata Martins dos Santos ${ }^{2}$
}

Odete Rocha ${ }^{3}$

RESUMO: Fipronil é um pesticida fenilpirazólico amplamente utilizado como defensivo agrícola nas culturas de cana-de açucar contra insetos-praga. O objetivo deste trabalho foi avaliar a toxicidade aguda do agrotóxico fipronil à biota de água doce, utilizando os rotíferos Asplanchna sieboldi, Brachionus caudatus e Lepadella patella como organismos-teste. Estes rotíferos foram obtidos de amostras de plâncton da Represa de Guarapiranga, situada em São Paulo, SP. Os testes de toxicidade foram realizados utilizandose o produto comercial Regente 800 WG $\AA$ cujo princípio ativo é o fipronil. Para $A$. sieboldi as concentrações testadas foram: 1,56, 3,12, 6,25 e 12,5 $\mathrm{mg} \mathrm{L}^{-1}$, para $B$. caudatus e $L$. patella 12,5, 25, 50 e $100 \mathrm{mg} \mathrm{L}^{-1}$. Os valores obtidos para a $\mathrm{CL}_{50}-48 \mathrm{~h}$ de fipronil foram: $10,65 \mathrm{mg} \mathrm{L}^{-1}$ para $A$. sieboldi e de 39,69 $\mathrm{mg} \mathrm{L}^{-1}$ para $B$. caudatus. $O$ rotífero $L$. patella não sobreviveu em nenhuma das concentrações testadas. Comparando-se as espécies, concluiu-se que $L$. patella foi a mais sensível ao fipronil e que $A$. sieboldi é menos sensível que $B$. caudatus. O importante papel que os rotíferos desempenham na comunidade

\footnotetext{
1 Biológa, Mestranda do Programa de Ecologia e Recursos Naturais, UFSCar - São Carlos. raquel.moreira87@yahoo.com.br

${ }^{2}$ Bióloga, Doutoranda em Ciências, Programa de Pós-Graduação em Ecologia e Recursos Naturais, UFSCar - São Carlos.

${ }^{3}$ Docente do Departamento de Ecologia e Biologia Evolutiva, Universidade Federal de São Carlos, UFSCar. doro@ufscar.br
} 
zooplanctônica dos corpos de água tropicais tornam imprescindíveis a realização de estudos que abordem aspectos biológicos e ecotoxicológicos em seus ciclos de vida, os quais são ainda incipientes. $O$ fato das espécies estudadas apresentarem alta sensibilidade, além de possuirem elevada taxa reprodutiva e curta longevidade, sugere que espécies deste grupo poderão ser uma ferramenta importante como organismosteste na avaliação da toxicidade deste e de outros agrotóxicos, visando a obtenção de subsídios para ações voltadas à preservação da diversidade da biota aquática.

Palavras-chave: Rotífero. Fipronil. Toxicidade.

\section{INTRODUÇÃO}

\section{Água como recurso natural}

Todas as formas de vida na Terra dependem da água. Apesar de $75 \%$ da superfície do globo ser recoberta por água, mais de $97 \%$ deste total é de água salgada, indisponível para o consumo direto e para a maioria dos usos pelo ser humano. Lagos e rios são as principais fontes de água potável, mesmo constituindo, em seu conjunto, menos de $0,01 \%$ do total de água do planeta (Baird, 2002). As atividades antropogênicas podem causar modificações ambientais resultando em mudanças na estrutura das comunidades e consequentemente no funcionamento dos ecossistemas. Entre os ecossistemas do planeta, os aquáticos constituem receptores temporários ou finais de uma grande quantidade e variedade de poluentes, sejam eles lançados ao ar, ao solo, ou diretamente nos corpos de água. Quando atingem os corpos de água, estes poluentes podem ter vários destinos, que dependem das propriedades físicas do composto, das características químicas, físicas e biológicas do ecossistema atingido, bem como da taxa de entrada do composto no ambiente (Rand, 1995).

\section{Pesticidas e sua relação com o ambiente aquático}

Segundo Ayibotele (1992), cerca de $70 \%$ da água consumida no mundo é utilizada para agricultura, $22 \%$ nos processos industriais e $8 \%$ para o consumo doméstico. Sendo 
assim, grande importância deve ser dada aos resíduos gerados pela atividade agrícola e suas consequências para os ecossistemas adjacentes.

Os agrotóxicos, entre eles os utilizados na agricultura, são compostos que podem matar diretamente um organismo indesejável ou controlá-lo de alguma maneira. Atualmente eles são massivamente utilizados na agricultura para que possam ser cultivadas grandes quantidades de determinadas espécies em uma pequena área, sem a interferência de organismos que possam reduzir a produtividade. Os fabricantes de agrotóxicos no mundo todo tem buscado o aumento da margem de segurança entre a toxicidade para os invertebrados alvo e a proteção da saúde humana. No entanto, estes compostos podem exercer efeitos em organismos diferentes dos alvos da aplicação, 0 que pode alterar o equilíbrio dinâmico de um ecossistema. Segundo Pimentel e Levitam (1991), em uma revisão sobre a quantidade de pesticidas aplicados, apenas $0,1 \%$ do produto, em média, atinge os organismos-alvo. O restante acaba por contaminar os ecossistemas adjacentes ao da aplicação. Tendo em vista que o ambiente aquático exerce um papel de receptor intermediário ou final de grande quantidade de compostos, quando há grande proximidade entre um agroecossistema e ambientes aquáticos, os riscos de contaminação são eminentes (Rand, 1995).

\section{A atuação do fipronil}

Nos ecossistemas aquáticos, os pesticidas podem ter diversos destinos: permanecerem dissolvidos na coluna d'água, alcançando o sedimento ou se acumulando nos tecidos dos organismos. O fipronil (5-amino-1-[2,6-dichloro-4-(trifluoromethyl)phenyl][(trifluoromethyl)sulfinyl]-1H-pyrazole-3-carbonitrile) é um inseticida fenil pirazólico, considerado um pesticida seletivo para insetos. Ele atua de forma semelhante aos inseticidas ciclodienos, como o endossulfan e o dieldrin interferindo na passagem de íons cloreto pelos canais de cloro dos neurônios, processo que é regulado pelo ácido gamaaminobutírico (GABA). Portanto, o fipronil atua no sistema nervoso, paralisando ou matando o organismo-alvo (Rhône-Poulenc,1998). Desta forma, ele tem sido amplamente utilizado para o controle de formas adultas e larvais de pragas, como baratas, mosquitos, moscas, besouros, etc., e particularmente em plantações de soja e cana-de-açúcar, para 
o controle de formigas, cupins e larvas de coleópteros. A ampla disseminação deste composto deve-se à sua eficácia no combate a insetos resistentes a outros pesticidas (Bobe et al., 1997). No Brasil, o início da utilização de fipronil foi registrado no ano 2000 em plantações da região Nordeste (Masutti e Mermut, 2007). No país, o produto Regent 800 WG, com 80\% de fipronil em sua composição, é aplicado em plantações de cana-deaçúcar no período entre junho e agosto, logo após o plantio, em sulcos no solo, com a finalidade de formar uma barreira contra insetos na subsuperfície sendo recomendada pelo fabricante a utilização de concentrações que variam de $200 \mathrm{~g}$ a $500 \mathrm{~g} \mathrm{ha}^{-1}$, dependendo do inseto a ser controlado.

Dados oriundos de diversos estudos demonstram que a maior concentração de fipronil registrada em águas naturais adjacentes a campos de agricultura foi de aproximadamente $0,12 \mu \mathrm{g} \mathrm{L}^{-1}$ no estado de Louisiana nos Estados Unidos (Gunasekara e Troung, 2007). No entanto, as características físico-químicas do composto demonstram que há uma partição considerável do fipronil entre a água e o sedimento representada pelo seu coeficiente de partição octanol-água (log Kow $=4,0)$ e que o sedimento representa um compartimento importante para a avaliação das concentrações de fipronil em um ecossistema aquático.

Segundo Beeler et al. (2001), os produtos de degradação do fipronil podem ser ainda mais tóxicos que o composto original. Em ordem crescente de toxicidade encontram-se: fipronil amida, fipronil e fipronil desulfinil, fipronil sulfona e o mais tóxico dos derivados, o fipronil sulfeto.

\section{Rotíferos como organismos-teste}

Os rotíferos são considerados organismos oportunistas, mostrando alta capacidade adaptativa, colonizando rapidamente uma grande variedade de habitats e nichos. Ocorrem preferencialmente em ecossistemas aquáticos continentais, apresentando grande diversidade de formas (Schäefer 1985). Eles constituem um elo importante na cadeia alimentar entre os produtores primários e consumidores secundários, tais como larvas de peixes e bentos (Nogrady et al., 1993). O papel dos rotíferos no fluxo de energia é de grande importância devido ao seu alto potencial reprodutivo associado com os seus 
ciclos de vida relativamente curtos, implicando uma conversão importante de matéria orgânica via produção secundária (Winberg, 1971; Elser et al, 1988). Os rotíferos também participam na reciclagem de nutrientes, e assim, na produtividade dos ecossistemas de água doce. De acordo com Makarewicz e Likens (1979), os rotíferos foram responsáveis por cerca de um terço do fósforo anualmente incorporado pelo zooplâncton em Mirror Lake (EUA).

Organismos deste grupo são amplamente utilizados na aquicultura como alimento para formas jovens de peixes e crustáceos (Neumann-Leitão \& Souza 1987) e destacamse também como bioindicadores das condições tróficas das águas (Berzins \& Pejler 1989, Güntzel \& Rocha 1998, Duggan et al. 2001, Nogueira 2001).

O objetivo do presente trabalho foi avaliar a toxicidade aguda do agrotóxico fipronil à biota de água doce, utilizando os rotíferos Asplanchna sieboldi, Brachionus caudatus e Lepadella patella como organismos-teste.

\section{MATERIAIS E MÉTODOS}

\section{Os Rotíferos Utilizados como Organismos-Teste}

Os rotíferos do gênero Asplanchna são representados por fêmeas com o corpo em forma de saco e desprovidas de pé. O macho manifesta um dimorfismo sexual bastante acentuado, com tamanho e morfologia distintos dos de uma fêmea. Possui projeções ou corcovas bem salientes na parede corporal, sendo duas laterais e uma no dorso. Apresenta apenas vestígios do aparelho digestivo, o qual se degenera durante 0 desenvolvimento embrionário.

O gênero Brachionus constitui um importante componente dos organismos planctônicos nos ecossistemas aquáticos, tendo um papel relevante em diversas pesquisas sobre a biota aquática. A possibilidade de cultivar estes organismos em laboratório tem permitido a realização de uma variedade de trabalhos científicos sobre algumas espécies do gênero. Espécies deste gênero estão bem representadas em diferentes corpos d'água em todo o mundo. Estudos realizados por Bucka (1968) 
mostraram o predomínio destes em corpos de água altamente eutróficos destacando-se os ambientes onde as condições são severas para os organismos de outros grupos.

Lepadella patella é um rotífero Monogononta, de maior ocorrência na zona litoral dos corpos d'água (Koste, 1978). Os gêneros Brachionus, Lecane, Lepadella e Trichocerca, pertencentes às famílias Brachionidae, Lecanidae, Lepadellidae e Trichocercidae, respectivamente, apresentam um grande número de espécies na fauna tropical de rotíferos, como já enfatizado por muitos autores (Paggi, 2001; Chittapun \& Pholpunthin, 2001; Sanoamuang \& Savatenalinton, 2001).

\section{Locais de origem dos rotíferos}

Amostras de água da Represa de Guarapiranga (Fig 1), situada na Área Metropolitana de São Paulo, SP (2343'S e 4632'W) foram analisadas em microscópio visando o isolamento dos rotíferos.

Concomitantemente à introdução dos organismos para meios de cultivo foram realizados ensaios ecotoxicológicos, visando dessa forma que sejam avaliadas e comparadas as respostas dos organismos quando expostos às diferentes concentrações testadas para o agrotóxico fipronil, por duas perspectivas, imediatamente após de terem sido retirados de seu ambiente natural e após a aclimatação do organismo em cultivo laboratorial.

De acordo com Esteves (1998) a associação dos rotíferos ao fitoplâncton é de tal maneira acentuada que nos casos de floração de algas ocorre o crescimento acentuado da população destes e, com o término da floração, os rotíferos desaparecem totalmente. Ferenska e Lewkovicz (1966) observaram um grande aumento no número de espécies de rotíferos, considerando-os indicadores de corpos de água eutróficos. A represa de Guarapiranga é caracterizada como um ambiente eutrófico, tendo ampla ocorrência de rotíferos em todo o seu corpo d'água. 


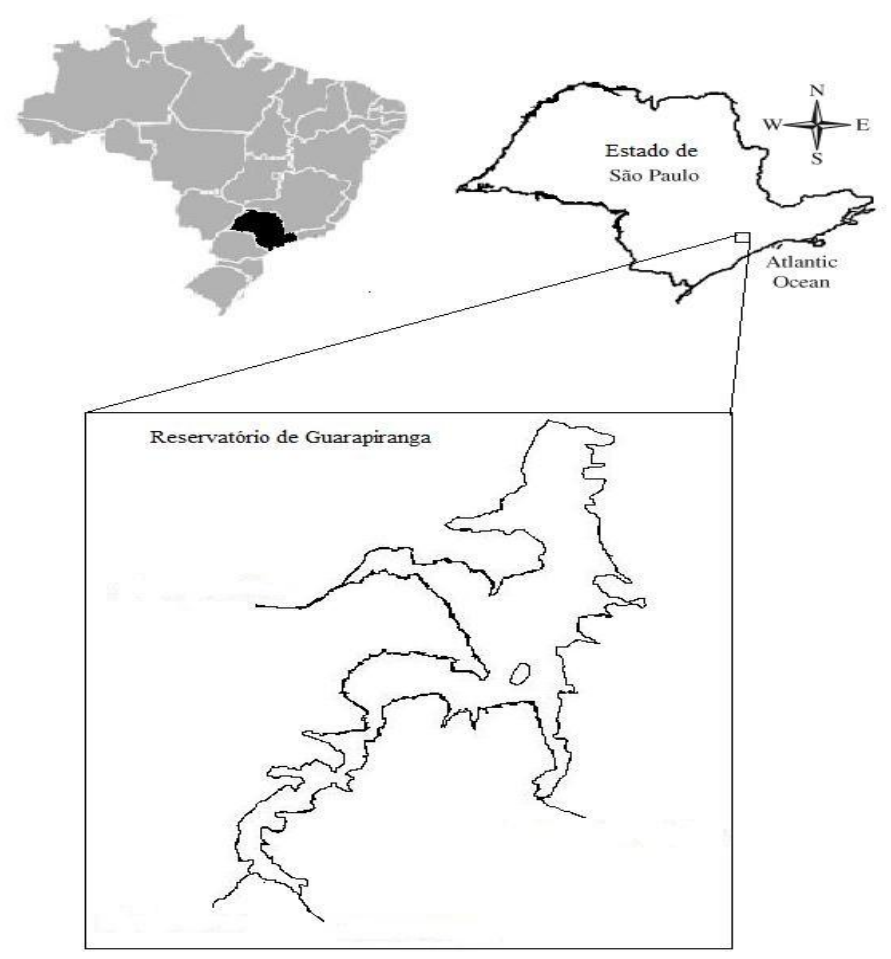

Fig. 1 Localização da Represa de Guarapiranga, situada na Área Metropolitana de São Paulo, SP (2343'S e 46ํㅜ' W), ambiente caracterizado como eutrófico (Sendacz et al.2006).

\section{Testes de toxicidade aguda com o agrotóxico fipronil}

Os testes de toxicidade aguda foram feitos utilizando-se o produto comercial Regente 800 WG ${ }^{\circledR}(80 \%$ fipronil e $20 \%$ celulose) como fonte de fipronil. A solução estoque foi preparada na concentração de $1 \mathrm{~g} \mathrm{~L}^{-1}$ diluído em água destilada.

Esta solução foi mantida em frasco totalmente escuro e em freezer comum $\left(-15^{\circ} \mathrm{C}\right)$ com o intuito de evitar a degradação do agroquímico antes de serem preparadas as diluições desejadas.

Nas cavidades de placas de poliestireno (Costar $\AA$ ) com 24 cavidades, usualmente utilizadas para cultivo de tecido, foram adicionadas a solução controle e as soluções-teste (1,56 mg L $\left.\mathrm{m}^{-1}, 3,12 \mathrm{mg} \mathrm{L}^{-1}, 6,25 \mathrm{mg} \mathrm{L}^{-1}, 12,5 \mathrm{mg} \mathrm{L}^{-1}\right)$ com 4 repetições para cada uma das concentrações. Foram utilizados dez organismos da espécie Asplanchna sieboldi em cada cavidade da placa de cultivo, colocados aleatoriamente em todas as cavidades. Em 
seguida as placas de cultivo com os organismos foram mantidos em incubadora em condições constantes de $24 \pm 1{ }^{\circ} \mathrm{C}$ de temperatura e de 16 horas luz/8 horas escuro de fotoperíodo durante um período de 48 horas de exposição ao agrotóxico.

As espécies Brachionus caudatus e Lepadella patella foram expostas a concentrações maiores do que a espécie Asplanchna sieboldi, com o intuito de ser avaliada uma faixa de sensibilidade mais ampla. As concentrações testadas foram: 12,5 $\mathrm{mg} \mathrm{L}^{-1}, 25,00 \mathrm{mg} \mathrm{L}^{-1}, 50,00 \mathrm{mg} \mathrm{L}^{-1}$ e $100,00 \mathrm{mg} \mathrm{L}^{-1}$.

Os dados obtidos foram analisados pelo o programa estatístico Trimmed Spearmann-Karber (TSK), para estimar as concentrações medianas letais do agrotóxico fipronil. (Hamilton et al., 1977), com intervalo de confiança de 95\% (Cls).

\section{RESULTADOS E DISCUSSÃO}

A $\mathrm{CL}_{50}$ - 48h do fipronil para Asplanchna sieboldi foi de $10,65 \mathrm{mg} \mathrm{L}^{-1}$ e para Brachionus caudatus de $39,69 \mathrm{mg} \mathrm{L}^{-1}$ (Fig 2). A espécie Lepadella patella não sobreviveu em nenhuma das concentrações testadas. Comparando-se as espécies estudadas, observou-se que $L$. patella foi a mais sensível e que $A$. sieboldi foi menos sensível que $B$. caudatus.
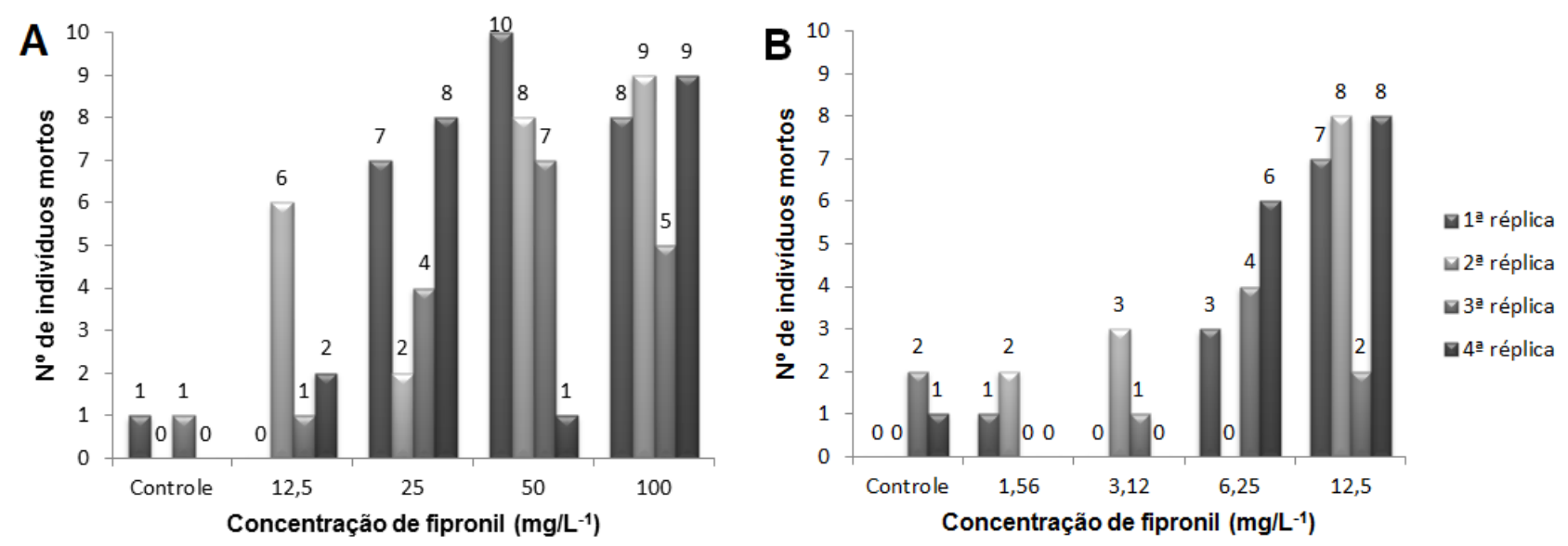

Fig. 2 Efeito tóxico de diferentes concentrações de fipronil para as espécies de Rotifera a): Asplanchna sieboldi e b) Brachionus caudatus após $48 \mathrm{~h}$ de exposição. 
Diversos artigos relatam que o fipronil é um composto pouco tóxico para peixes e moluscos (Wirth et al., 2004) e altamente seletivo para insetos, absorvido por estes organismos primariamente via ingestão (Chaton et al, 2001).

Gunasekara e Troung (2007) em uma revisão sobre o destino ambiental do fipronil, destacaram a toxicidade do composto para insetos, avaliada pela CL50 48h, que variou de 0,4 a $23 \mu \mathrm{g} \mathrm{L}^{-1}$. Estes valores são extremamente baixos, quando se compara com os efeitos em outros organismos aquáticos, como crustáceos e moluscos, o que nos levaria a crer que entre os invertebrados, quanto mais desenvolvido o sistema nervoso do organismo, mais sensível seria ao fipronil, devido à sua neurotoxicidade (Manrique, 2009).

Segundo Rhône-Poulenc, 1996, o fipronil atua de forma semelhante aos inseticidas ciclodienos, como endossulfan e dieldrin, interferindo na passagem de íons cloreto pelos canais de cloro dos neurônios, processo que é regulado pelo ácido gamaaminobutírico (GABA). No Brasil, o Conselho Nacional para a Meio Ambiente (CONAMA 2005) recomenda um nível máximo de Aldrin + Dieldrin de $0,005 \mu \mathrm{g} \mathrm{L}^{-1}$ e de Endossulfan $(\alpha+\beta$ + sulfato) ${ }^{67}$ de $0,056 \mu \mathrm{g} \mathrm{L}^{-1}$ em águas de superfície, a fim de proteger a saúde humana e a vida aquática. Já a Comunidade Européia estabeleceu padrões de potabilidade para águas destinadas ao consumo humano de $0,1 \mu \mathrm{g} \mathrm{L}^{-1}$ para um e $0,5 \mu \mathrm{g} \mathrm{L}^{-1}$ para a soma de todos os agrotóxicos presentes, incluindo seus metabólitos (EC - European Community, 1998). Com os resultados de $\mathrm{CL}_{50}$ - $48 \mathrm{~h}$ do pesticida fipronil obtidos no presente estudo para os rotíferos Asplanchna sieboldi e Brachionus caudatus, essas espécies aparentemente estariam protegidas dos efeitos agudos e crônicos deste agrotóxico. No entanto essa afirmação pode não ser verdadeira, levando em consideração que as concentrações reais encontradas em ambientes lóticos e lênticos poderão estar acima do nível máximo permitido por lei, como detectado na sub-bacia do rio Corumbataí (bacia do rio Piracicaba) para o agrotóxico atrazina. Esta é uma região amplamente ocupada por plantações de cana-de-açúcar, em que as concentrações de atrazina variaram entre 0,6 e 2,7 $\mathrm{Mg} \mathrm{L}^{-1}$, valores encontrados durante o verão chuvoso (Armas et al. 2007). O Conselho Nacional para a Meio Ambiente (CONAMA 2005) recomenda um nível máximo de atrazina de $2 \mu \mathrm{g} \mathrm{L}^{-1}$ em águas de superfície.

A Tabela 1 mostra uma classificação dos agrotóxicos, em relação às suas classes de toxicidade, de acordo com Zucker (1985). De acordo com este quadro, o fipronil pode 
ser considerado ligeiramente tóxico para Asplanchna sieboldi e Brachionus caudatus segundo os resultados obtidos neste trabalho.

Tabela 1 - Classes de toxicidade aguda de agrotóxicos para organismos aquáticos.

\begin{tabular}{lc}
\hline Classe de toxicidade & CL50 ou CE50 (mg.L') \\
\hline Extremamente tóxico & $<0,1$ \\
Altamente tóxico & $0,1 \mathrm{a} 1,0$ \\
Moderadamente tóxico & $>1,0 \mathrm{a}<10$ \\
Ligeiramente tóxico & $>10 \mathrm{a}<100$ \\
Praticamente năo-tóxico & $>100$ \\
\hline
\end{tabular}

Fonte: Zucker (1985)

Os resultados obtidos no presente trabalho são preliminares, mas promissores tendo em vista a sensibilidade observada para as espécies utilizadas como organismosteste. Além disso, levando-se em consideração a provável capacidade dos rotíferos de bioacumular e transferir o fipronil para os níveis tróficos superiores, o que afetaria indiretamente a biota do sistema aquático.

\section{CONCLUSÃO}

O relevante papel desempenhado pelos rotíferos nos corpos de água tropicais tornam imprescindível a realização de estudos que abordem seus aspectos biológicos e ecotoxicológicos, os quais são ainda incipientes. O fato das espécies Lepadella patella, Asplanchna sieboldi e Brachionus caudatus apresentarem alta sensibilidade ao pesticida testado, além de possuirem uma taxa reprodutiva proporcionalmente alta e longevidade relativamente curta, sugere que as espécies deste grupo poderão ser organismos-teste bastante úteis.

Estas espécies poderão se tornar uma ferramenta útil na avaliação da toxicidade deste e de outros agrotóxicos, visando a obtenção de subsídios para ações voltadas à preservação da diversidade da biota aquática. 


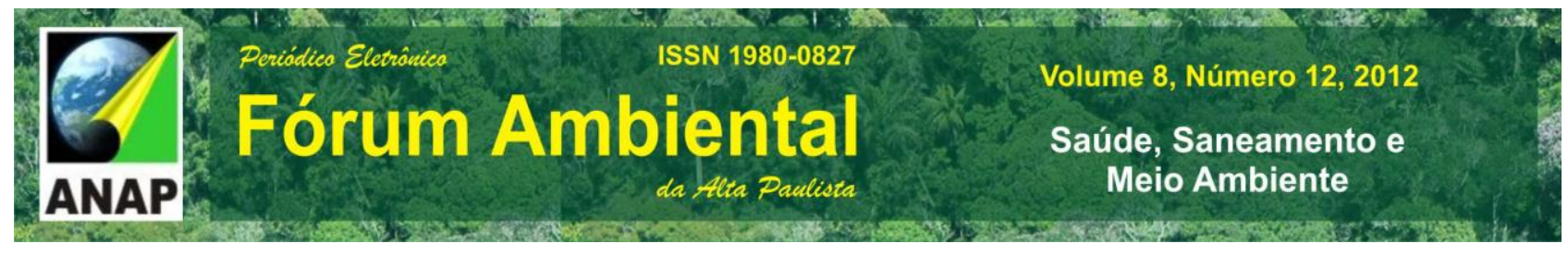

\section{REFERÊNCIAS}

ARMAS ED, ROSIM-MONTEIRO RT, AMÂNCIO AV, CORREA RML, GUERCIO MA. 2005. Uso de agrotóxicos em cana-de-açúcar na bacia do Rio Corumbataí e o risco de poluição hídrica. Quím Nova 28(6):975-982.

AYIBOTELE, N. 1992. The world's Water: Assessing the resource. International conference on Water and Environment: Development issues for the $21^{\text {th }}$ century. 26-31 de Janeiro, Dublin, Irlanda.

BAIRD, C. 2002. Química Ambiental. $2^{\circ}$ Edição. Porto Alegre. Editora Bookman, 622p.

BEELER, A.; SCHLENK, D.K. 2001. Synthesis of fipronil Sulfide, an Active Metabolite, From the Parent Insecticide fipronil. TETRAEDRON LETTERS 42(32) 5371-5372.

BERZINS, B. \& PEJLER, B. 1989. Rotifer ocurrence in relation to oxygen content. Hydrobiologia. 183:165-172.

BOBE, A., COOPER, J-F., COSTE, C.M. 1997. Factors Influencing the adsorption of fipronil on soils. Journal of Agriculture and Food Chemistry. 45 4861-4865.

BUCKA, H. 1968. Plankton communities in the Ochaby complex of experimental fishery farms, Acta Hidrobiol., 8(1):13-46.

CHATON, P.F.; RAVANEL, P.; TISSUT, M.; MEYRAN, J.C. 2002. Toxicity and bioaccumulation of fipronil in the non-target Arthropodan fauna associated with subalpine mosquito breeding sites. Ecotoxicology and Environmental Safety 52 08-12.

CHITTAPUN, S.; PHOLPUNTHIN, P. 2001. The rotifer fauna of peat-swamps in Southern Thailand. Hydrobiologia, v. 446/447, p. 255-259, 2001.

CONAMA. 2005. Dispõe sobre a classificação dos corpos de água e diretrizes ambientais para o seu enquadramento, bem como estabelece as condições e padrões de lançamento de efluentes, e dá outras providências. Resolução CONAMA no 357, de 17 de março de 2005.

DUGGAN, I.C., GREEN, J.D., SHIEL, R.J. 2001. Distribution of rotifers in North island, New Zealand, and their potential use as indicator of lake trophic state. Hydrobiologia 446:155-164.

EC - European Community; Council Directive 98/83/EC of 1998 on the quality of water intended for human consumption, Official Journal L 330, 05/12/1998, p. 0032-0054. Community legislation in force - document 398L0083 (1998). 
ELSER, J. J., ELSER, M. M., MACKAY, N. A. \& CARPENTER, S. R. 1988. Zooplanktonmediated transitions between $N$ - and P-limited algal growth. Limnology and Oceanography, 33(1): 1-14.

ESTEVES, F.A. 1998. Fundamentos de Limnologia. 2.ed. Rio de Janeiro: Interciência, Finep.

FERENSKA, M.; LEWKOVICZ, S. 1966. Zooplankton in ponds in relation to certain chemical factors. Acta Hidrobiol. 8(1):127-153.

GUNASEKARA AS; TRUONG T; GOH KS; SPURLOCK F; TJEERDEMA RS. 2007. Environmental fate and toxicology of fipronil. J Pestic Sci 32:189-199.

GÜNTZEL, A. \& ROCHA, O. 1998. Relações entre a comunidade zooplanctônica e as condições tróficas da lagoa Caconde, Osório, RS, Brasil. Iheringia, Sér. Zool. 84:65-71.

Hamilton MA, Russo RC, Thurfton RB. 1977. Trimmed Spearman-Karber method for estimating median lethal concentration in toxicity bioassays. Environ Sci Technol 11(7):714-719

Koste, W., 1978. Rotatoria. Die Rädertiere Mitteleuropas. Ein Bestimmungswerk begründet von Max Voigt. Bornträger, Stuttgart.Vol. 1: Textband 673 pp., Vol. 2: Tafelband $234 \mathrm{pp}$.

MAKAREWICZ, J. C. \& LIKENS, G. E. 1979. Structure and function of the zooplankton community of Mirror Lake, New Hampshire. Ecological Monographs, 49(1): 109-127.

MANRIQUE, W. G. 2009. Toxicidade aguda e risco ecotoxicológico do fipronil para o guaru (Poecilia reticulata) e dissipação no ambiente aquático. Universidade Estadual Paulista - Jaboticabal/SP, 58p.

MASUTI, C.S.M; MERMUT, A.R. 2007. Degradation of friponil under laboratory conditions in a tropical soil from Sirinhaém Pernambuco, Brazil. Journal of Environmental Science and Health Part B. 42, 33-43.

MASUTI, C.S.M; MERMUT, A.R. 2007. Sorption of fipronil and its sulfide derivative by soils and goethite. Geoderma 140 (1-2) 01-07.

PENNAK, R.W. 1953. Fresh-water invertebrates of the United States. New York: The Ronald Press Company, $769 \mathrm{p}$.

NEUMANN-LEITÃO, S. \& SOUZA, F.B.V.A. 1987. Rotíferos planctônicos do açude de Apipucos. Recife-PE (Brasil). Arq. Biol. Tecnol. (Braz. J. Biol.) 30(3):393-418. 
NOGRADY, T., WALLACE, R. L. \& SNELL, T. W., 1993. Rotifera. Volume 1: Biology, ecology and systematics. Guides to the identification of the micro-invertebrates of the continental waters of the world. SPB Academic Publishing, The Hague, 142p.

NOGUEIRA, M.G. 2001. Zooplankton composition, dominance and abundance as indicators of environmental compartimentalization in Jurumirim reservoir (Paranapanema river), São Paulo, Brazil. Hydrobiologia. 455:1-18.

PAGGI, S. J. 1995. Rotiferos - aspectos metodologicos. Workshop Invertebrados de água doce neotropicais.

PIMENTEL \& LEVITAM. 1986. Pesticide: amounts applied and amounts reaching plants. BioScience, 36:86-91.

RAND, G.M. 1995. Fundamentals of Aquatic Toxicology: effects, environmental fate and risk assessment. New York-NY. Taylor e Francis, 1124 p.

RHÔNE-POULENC AG COMPANY. 1998. Application for Registration. Volume No. 52062-071. Vol 1. Department of pesticide regulation, California Environmental Protection Agency, Sacramento, CA.

SANOAMUANG, L.; SAVATENALINTON, S. 2001. The rotifer fauna of Lake Kud-Thing, a shallow lake in Nong Khai Province, northeast Thailand. Hydrobiologia, v. 446-447, p. 297304.

SCHÄEFER, A. 1985. Fundamentos de ecologia e biogeografia de águas continentais. Editora da UFRGS, Porto Alegre.

SENDACZ, S., CALEFFI, S. and SANTOS-SOARES, J., 2006. Zooplankton biomass of reservoirs in different trophic conditions in the state of São Paulo, Brazil. Revista Brasileira de Biologia = Brazilian Journal of Biology, vol. 66, no. 1B, p. 337-350.

WINBERG, G. G., 1971. Methods for estimating the production of populations with continuous reproduction. In: G. G. Winberg, Methods for the estimation of production of aquatic animals. Translated from the Russian by A. Duncan. Royal Holloway College, University of London, London.

WIRTH, E.F.; PENNINGTON, P.L., LAWTON, J.C., DELORENZO, M.E., BEARDEN, D.; SHADDRIX, B., SIVERTSEN, S., FULTON, M.H. 2004. The Effects of contemporary-use insecticide (fipronil) in an estuarine mesocosm. Environmental Pollution 131: 365-371.

ZUCKER, E. 1985. Hazard Evaluation Division - Standard Evaluation Procedure Acute toxicity test for freshwater fish. (USEPA Publication 540/9-85-006). 